\title{
Attentional artifacts in sensorimotor coupling in the postural control of young adults
}

\author{
Giovanna Gracioli Genoves ${ }^{1,2}$ - Ana Maria Forti Barela ${ }^{2}$ Caroline Sanches ${ }^{1}$. \\ José Angelo Barela ${ }^{1,2}$
}

Received: 23 December 2015 / Accepted: 18 August 2016 / Published online: 24 August 2016

(C) Springer-Verlag Berlin Heidelberg 2016

\begin{abstract}
Although postural control requires the integration of different sensory cues, little is known about the role of attentional artifacts on the individual's ability to properly respond to postural challenges. This study investigated the effects of concomitant tasks (cognitive and postural) on the relationship between visual information and body sway. Thirty healthy adults were asked to stand still inside of a moving room on normal and reduced bases of support. Initially, the participants were not aware of any visual manipulation and were asked to perform tasks that required concomitant attentional demands. Then, all participants were informed about the visual manipulation and were requested to resist it. The results showed that information about visual manipulation changed the coupling between visual information and body sway, but only in a less demanding task, and that it was affected by the concomitant task. The coupling between visual information and body sway for postural control does not demand attention on a regular basis, but any change in this relationship demands attention and occurs in less demanding postural tasks.
\end{abstract}

Keywords Dual-task · Attention · Sensorimotor coupling · Task demand $\cdot$ Posture

José Angelo Barela

jbarela@rc.unesp.br

1 Institute of Biosciences, São Paulo State University, Rio Claro, SP, Brazil

2 Institute of Physical Activity and Sport Sciences, Cruzeiro do Sul University, São Paulo, SP, Brazil

\section{Introduction}

The human postural control system is quite complex. To continuously perform a simple and basic motor task, such as maintaining an upright stance, the central nervous system (CNS) must accurately integrate sensory cues originating from different channels, such as visual, vestibular, and somatosensory (Nashner 1981). This integration process requires humans to converge information regarding body orientation as well as those coming from force vectors that may trigger the necessary muscle activity to maintain the body at the desired position (Horak and Macpherson 1996). Considering that sensory cues come from multiple channels and that multilinked muscles compose the effector system, the coupling between sensory information and muscle activity becomes a nontrivial task that greatly challenges the CNS. In addition, complexity inherently increases because the sensory-motor system must continuously respond to ever-changing environmental demands.

Schöner et al. (1998) proposed a strategy to examine the relationship between sensory information and motor action by directly manipulating sensory cues, while all the other sources of sensory information remained unaltered. This strategy has been widely explored to examine the use of visual (Barela et al. 2009, 2014; Stoffregen et al. 2006; Toledo and Barela 2014), somatosensory (Barela et al. 2003; Jeka et al. 1998), and visual and somatosensory cues simultaneously (Polastri et al. 2012; Viana et al. 2013) to uncover critical correlates of human postural control. An interesting common finding from these studies was that humans were able to continually couple different sensory systems without consciously noticing the manipulation of small amplitude and low velocity sensory cues. Interestingly, when these individuals were informed and/or requested to resist to it, the amplitude of their body sway 
was significantly reduced (Barela et al. 2014; Freitas Junior and Barela 2004; Stoffregen et al. 2006).

Human postural control has also been known to be task dependent (Horak and Macpherson 1996). For example, when the body's base of support (foot position) is reduced, the magnitude of the body sway dramatically increases (Prioli et al. 2005, 2006; Streepey and Angulo-Kinzler 2002). One reasonable explanation is that refined and more accurate levels of sensory integration may be required to perform tasks that challenge the limits of the postural control system (Prioli et al. 2006).

Although it has been shown that the manipulation of sensory information (without conscious effort) induces body sway and that more challenging postures demand refined sensory coupling strategies between the sensory and motor systems, little is known about the role of attentional artifacts on the individual's ability to control their own posture. Several studies have investigated the role of attention and its relationship between sensory information and motor action (Andersson et al. 2002; Remaud et al. 2012; Siu et al. 2008). Overall, the strategy adopted by these researchers was the dual-task approach, in which participants were asked to maintain an upright stance while they concomitantly performed a second task that demanded cognitive efforts, such as reading (Bucci et al. 2015), visual searching (Prado et al. 2007), and counting backwards (Aguiar et al. 2014). Surprisingly, the performance of the postural control system decreased, which suggested that postural adjustments typically rely on attentional resources (Andersson et al. 2002). More recently, Aguiar et al. (2014) observed that if individuals were aware that visual cues are being manipulated, they significantly reduced postural sway, and as they were asked to resist it, they reduced it even more. Unexpectedly, another interesting finding in that study was that postural control effects did not differ when researchers compared an experimental condition that participants were not aware of the visual cue manipulation to a condition that participants were asked to count backwards and resist the manipulation of the visual cue (a tri-task). Altogether, these findings suggest that sensory-motor coupling demands attention and cognitive efforts (Aguiar et al. 2014). Therefore, attentional demand seems to be a critical factor that alters the coupling between sensory information and motor action in postural control.

To further explore this issue, we designed an experiment to test whether any change in the relationship between sensory information and body sway would demand more attentional resources. In addition, we also tested whether such demand would be different when participants were asked to perform a task that challenged their postural control. Therefore, this study examined the effects of concomitant tasks (cognitive and postural) on the relationship between visual information and body sway.

\section{Methods}

\section{Participants}

Thirty young healthy adults with no reported history of vestibular or neurologic disorders were equally divided into two task groups (reduced and normal base of support). Seven males and eight females were assigned to each group according to their age $(22 \pm 2.3$ and $21.3 \pm 1.85$ years old), body mass $(64.4 \pm 10.1$ and $65.7 \pm 12.1 \mathrm{~kg})$, and height $(1.67 \pm 0.08$ and $1.67 \pm 0.01 \mathrm{~m})$. The experiment was performed with the written consent of each participant. All experimental procedures were reviewed and approved by the institutional ethics committee.

\section{Procedures}

All participants were asked to maintain an upright and quiet stance inside a moving room and to look straight ahead at a target $(5 \mathrm{~cm}$ of diameter) fixed on the front wall $1 \mathrm{~m}$ away. The moving room consisted of three walls and a roof $(2.1 \mathrm{~m}$ long $\times 2.1 \mathrm{~m}$ wide $\times 2.1 \mathrm{~m}$ height $)$, mounted on casters so that it could be moved back and forth by a servomotor mechanism, while the floor remained motionless. The walls and the roof were white, and black stripes were painted on the walls, creating a pattern of $42-\mathrm{cm}$-wide vertical white and $22-\mathrm{cm}$-wide vertical black stripes. A 20-W fluorescent lamp was attached to the ceiling to maintain consistent lighting throughout the test.

The room's movement was produced by a servomotor system composed of a stepper motor (Ottime, model SM23 SSF11921088) and a motor driver (Ottime, model MBD278AC) and controlled by specific routines (Motion Planner 4.3). All participants included in the "normal base of support" group were asked to stand on a force platform (Kirstler, model 9286A), with their feet parallel and waist width apart. All participants included in the "reduced base of support group" were asked to adopt the same position, but standing on a wood beam $(8 \mathrm{~cm}$ width $\times 2 \mathrm{~cm}$ height) placed on top of the force platform. This strategy was adopted to reduce their basis of support and to prevent them from touching the floor with their toes and heels (Oullier et al. 2004; Prioli et al. 2006). Participants from both groups were asked to stand as still as possible with their arms passively hanging beside their trunk.

Each participant performed nine trials of $60 \mathrm{~s}$ each. During the first trial, the room remained stationary. For all additional eight trials, the moving room oscillated back and forward at a peak velocity of $0.6 \mathrm{~cm} / \mathrm{s}$, peak-to-peak amplitude of $0.6 \mathrm{~cm}$ and at a frequency of $0.2 \mathrm{~Hz}$. These parameters were chosen based on previous studies (Barela et al. 2009; Freitas Junior and Barela 2004) to induce postural sway that was only noticeable when participants were 
informed about the visual manipulation. For the first block of moving trials (trials \# 2, 3, 4 and 5), no information about the room movement was provided. For the second block of moving trials (trials \# 6, 7, 8, and 9), participants were informed that the room would be oscillating and were asked to resist the room's movement (resisting condition). Randomly, in two trials from both blocks (no instruction and resisting conditions), the participants were requested to also mentally count backwards from 100 in steps of three $(100,97,94, \ldots)$, performing either a dual- or a tri-task: an upright stance with no instruction and counting (dualtask) or an upright stance, resisting the room's movement and counting (tri-task). Overall, four experimental conditions were performed by both the normal base of support and reduced base of support groups: (1) no instruction and not counting; (2) no instruction and counting; (3) resisting and not counting; and (4) resisting and counting.

Anterior-posterior room displacement data were obtained using an electrical goniometer (EMG System do Brasil). Data from the force platform and electrical goniometer were synchronized and acquired (sample frequency of $100 \mathrm{~Hz}$ ) with an analog data acquisition unit (ODAUII), part of the OPTOTRAK system (Northern Digital, Inc.).

\section{Data analysis}

From the components of ground reaction forces, the center of pressure (CP) for both the anterior-posterior (AP) and medial-lateral (ML) directions was calculated. Then, CP and the moving room position were filtered (second-order zero lag low-pass Butterworth-cutoff frequency of $5 \mathrm{~Hz}$ ). Because movement of the room occurred in the AP direction, the following analyses were performed only for this direction. Finally, the trial in which the room remained stationary was not included in the present analyses.

Results from the CP were used to indicate the magnitude of each participant's postural sway. A first-order polynomial was fitted to each trial and subtracted from each $\mathrm{CP}$ data point of the respective trial to exclude any shifting not related to the movement induced by the moving room. Then, a standard deviation from all values for the corresponding trial was calculated to indicate sway variability. The relationship between room movement and postural sway was examined by calculating the following variables: coherence, gain, and phase. Coherence was selected to indicate the strength between room movement and oscillation of the $\mathrm{CP}$ at the frequency of the driving signal (moving room, $0.2 \mathrm{~Hz}$ ). Coherence values close to one/zero indicated strong/weak dependency between these two signals. Gain and phase indicated the influence of the moving room on the oscillation magnitude of CP. Altogether, these variables indicated the coupling structure between body sway and visual information. These variables were calculated through a transfer function (frequency response function), which was computed by dividing the Fourier transforms of CP signals by the Fourier transforms of the driving signal (moving room), resulting in a complex-valued transfer function. Gain corresponded to the ratio between the amplitude of $\mathrm{CP}$ signals and visual stimulus at the driving frequency $(0.2 \mathrm{~Hz})$. Gain values of one indicated a response amplitude similar to the stimulus amplitude, and lower/higher values indicated that the response amplitude was lower/higher than the stimulus driving amplitude. Phase depicted the temporal relationship between the oscillation of $\mathrm{CP}$ and the visual stimulus. Positive (negative) phase values indicate that sway was ahead (behind) the visual stimulus. Finally, considering that two trials for each experimental condition were obtained and analyzed, values for each respective condition were averaged and used for further statistical analyses.

Two analyses of variance (ANOVAs) and one multivariate analysis of variance (MANOVA) were performed using group (normal and reduced bases of support) and condition (no information and not counting; no information and counting; resisting and not counting; and resisting and counting) as factors; repeated measures ANOVA was used for the former factor. The dependent variables for each ANOVA were mean sway amplitude and coherence. The dependent variables for the MANOVA were gain and phase. When necessary, univariate analyses and Tukey's HSD post hoc tests were used.

\section{Results}

Figure 1 depicts the mean sway amplitude for both groups in all experimental conditions. ANOVA revealed group $\left(F_{1,28}=73.08, p<0.001, \eta^{2}=0.723\right)$ and condition $\left(F_{3,84}=10.06, p<0.001, \eta^{2}=0.264\right)$ effects, but no group by condition interaction $\left(F_{3,84}=1.65, p>0.05\right.$, $\left.\eta^{2}=0.056\right)$. The magnitude of $\mathrm{CP}$ was larger for the reduced base of support group. Post hoc tests revealed that when aware of the room's movement, the participants reduced the magnitude of $\mathrm{CP}$. No difference was found when participants were asked to count backwards.

Figure 2 depicts coherence values for both groups in all conditions. ANOVA revealed group $\left(F_{1,28}=6.25\right.$, $\left.p<0.001, \eta^{2}=0.188\right)$ and condition $\left(F_{3,84}=6.07\right.$, $\left.p<0.001, \eta^{2}=0.178\right)$ effects and a group by condition interaction $\left(F_{3,84}=4.63, p<0.005, \eta^{2}=0.142\right)$. Post hoc tests revealed that coherence values were similar for both groups in the no instruction conditions. On the other hand, in the resist condition, coherence values were reduced for the normal base of support group. Coherence values did not differ between groups for the resist and counting conditions. 


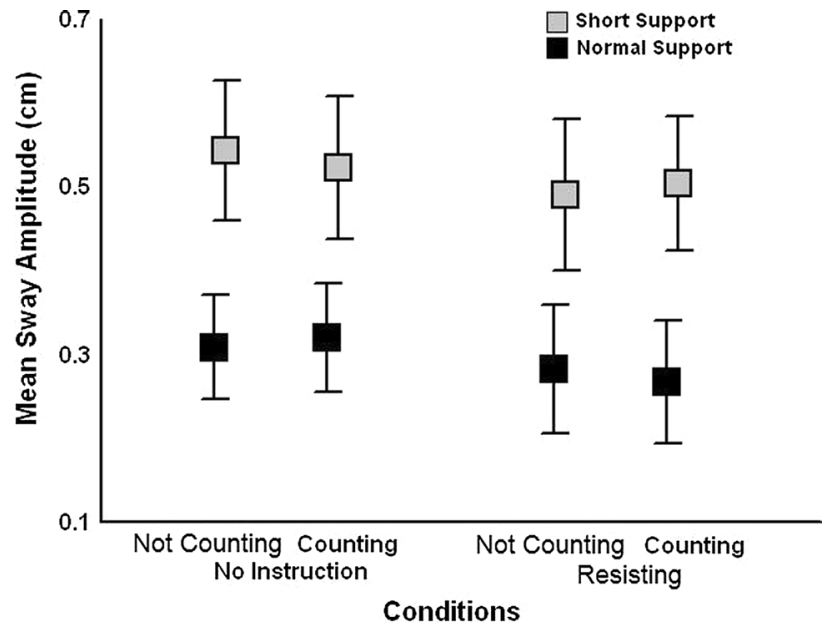

Fig. 1 Means $( \pm \mathrm{SD})$ values of mean sway amplitude for both groups (reduced and normal support) in all experimental conditions (no instruction and not counting; no instruction and counting; resisting and not counting; and resisting and counting)

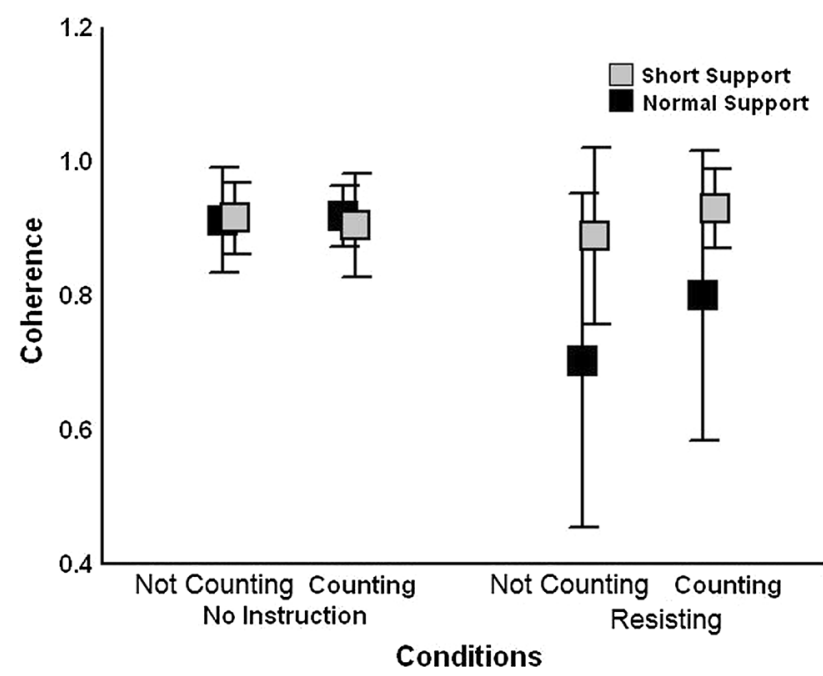

Fig. 2 Mean $( \pm \mathrm{SD})$ values for coherence for both groups (reduced and normal support) in all experimental conditions (no instruction and not counting; no instruction and counting; resisting and not counting; and resisting and counting)

Figure 3 depicts gain and phase values for both groups in all experimental conditions. MANOVA revealed group (Wilks' Lambda $=0.194, F_{2,27}=55.99, p<0.001$, $\eta^{2}=0.806$ ) and condition (Wilks' Lambda $=0.215$, $\left.F_{6,23}=14.02, p<0.001, \eta^{2}=0.785\right)$ effects, but no group by condition interaction $\left(F_{6,23}=1.71, p>0.05\right.$, $\left.\eta^{2}=0.309\right)$. Univariate analyses for both gain and phase revealed group $\left(F_{1,28}=67.14, p<0.001, \eta^{2}=0.706\right.$ and $F_{1,28}=17.97, p<0.001, \eta^{2}=0.391$, respectively) and condition $\left(F_{3,84}=12.68, p<0.001, \eta^{2}=0.312\right.$ and $F_{3,84}=3.93, p<0.001, \eta^{2}=0.123$, respectively) effects.

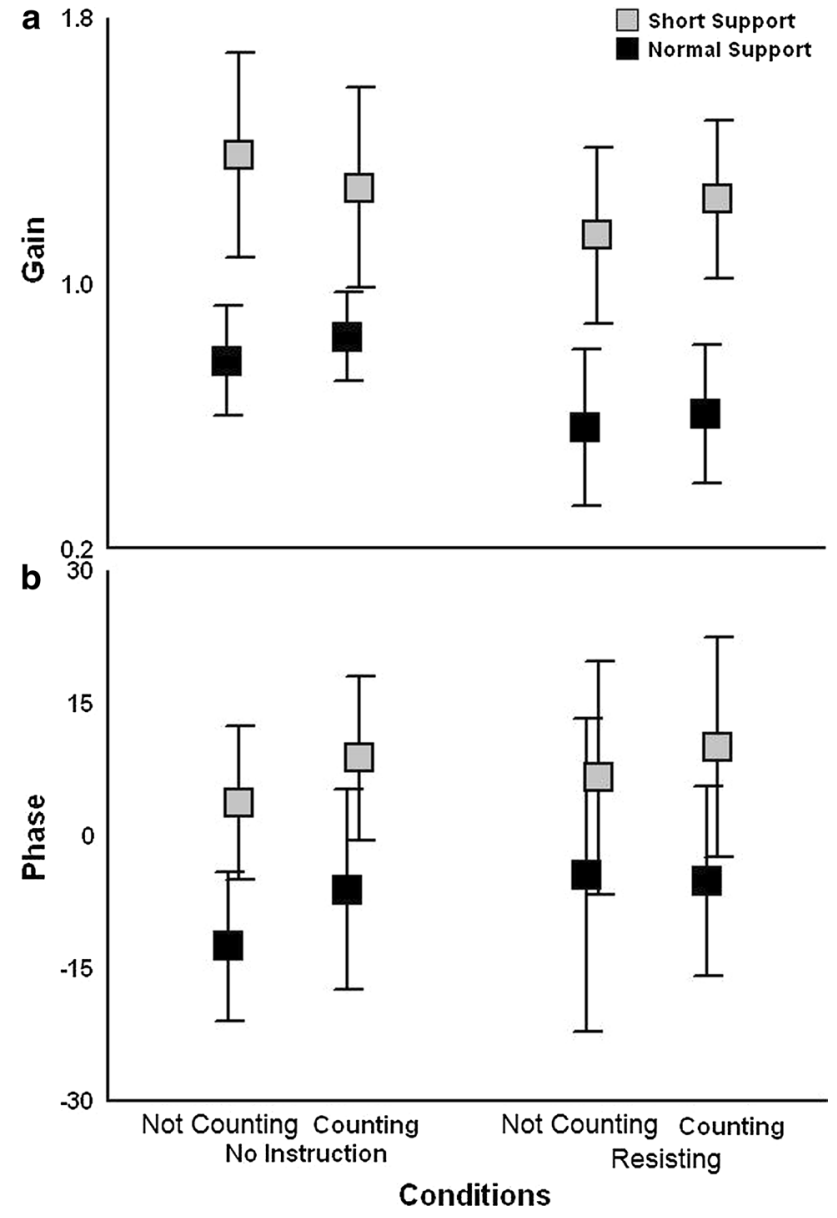

Fig. 3 Mean and $( \pm$ SD) values for gain (a) and phase (b) for both groups (reduced and normal support) in all experimental conditions (no instruction and not counting; no instruction and counting; resisting and not counting; and resisting and counting)

Gain values were lower for the normal base of support group, which indicated less influence of visual manipulation on postural control. Post hoc tests revealed that visual influence was reduced for both groups in the resist conditions. Phase values indicated that body oscillation was ahead of the room's movement for the normal base of support group and behind it for the reduced base of support group. Post hoc tests also revealed that the participants from the reduced base of support group were less ahead, and participants from the normal base of support were more behind in the no instruction and no counting conditions compared to all other conditions.

\section{Discussion}

This study aimed to examine the effects of concomitant tasks (cognitive and postural) on the relationship between visual information and body sway. Visual manipulation 
throughout the moving room induced corresponding postural sway in all participants. Larger body sway amplitudes were found on participants from the reduced base of support group. Interestingly, the task demand changed the temporal relationship between the moving room and body sway. Participants from the normal base of support group swayed slightly behind the moving room, and participants from the reduced base of support group swayed slightly ahead the moving room. When participants were informed about the visual manipulation and requested to resist it, they reduced the magnitude of body sway and the influence of the moving room on it, regardless of the postural task. In addition, although the induction of body sway was observed after the visual manipulation of both groups, body sway was less related to the moving room in those from the normal base of support group compared to those from the reduced base of support group. The concomitant task did not change the magnitude of body sway and visual influence when participants were not aware of the visual manipulation. On the other hand, when participants from the reduced base of support group were requested to avoid the influence of visual information and perform a third task (counting backwards), those participants swayed with the room as previously observed. Altogether, these findings suggest that when standing on a normal base of support, the coupling between visual information and body sway does not require many attentional resources. However, when standing on a reduced base of support, this coupling can only partially change and demands more of the individual's attention.

Visual manipulation through a moving room induced different magnitudes of body sway for different bases of support. Participants from the reduced base of support group presented larger magnitudes of body sway (Fig. 1) compared to the other group, indicating a greater influence of visual manipulation (Fig. 3), which is in accordance with previous findings (Prioli et al. 2006). Moreover, visual manipulation induced body sway in participants from both groups, even when they were unaware of such manipulation. Similarly to previous observations (Aguiar et al. 2014; Barela et al. 2014; Freitas Junior and Barela 2004; Stoffregen et al. 2006) in which participants stood on regular base of support, participants standing on a reduced base of support swayed coherently with the visual manipulation without being aware of such behavior, as observed when participants did not receive any instruction.

Although body sway was induced and occurred unconsciously, the participants from the reduced base of support group were more susceptible to the visual manipulation as they presented a larger body sway ahead of the moving room. Participants from the normal base of support group swayed behind the moving room (Fig. 3b). Such an observation is quite intriguing for the following reasons: Different bases of support certainly provide unique mechanical constraints (Oullier et al. 2004) allowing different mechanical supports for force production. Such a reduction of the base of support has been shown to constrain ankle-hip coordination, with adults adopting the same body segment relationship throughout different frequencies (Oullier et al. 2004), which suggested that the mechanical aspects overcome visual influences. These mechanical influences on a reduced base of support would induce larger body sway (as shown in this study), due to the smaller base of support for force and torque production. In this case, body sway would lag behind the moving room, since it would take longer to change the body's moment of inertia. Controversially, the results of this study revealed exactly the opposite; sway occurred ahead of visual manipulation.

We also expected that in a more demanding postural task, the coupling between sensory information and body sway would be stronger (Prioli et al. 2006) because the postural control system would produce more accurate and coherent motor activity to maintain postural orientation, despite the fact that it still can be modulated by intention (Oullier et al. 2002, 2004). However, our results suggest that the postural control system adopted a prospective strategy, as the participants swayed ahead of the moving room to overcome the mechanical constraints imposed by the reduced base of support. Moreover, the coupling strength was rather strong (coherence values close to 0.9, Fig. 2) and remained around this level across all experimental conditions presented to the reduced base of support group. Not only was the coupling between sensory information and body sway stronger in a more demanding postural task but also the strategy adopted by the postural control system may have changed to overcome task constraints. Such a different temporal strategy seems to be accomplished by adopting different types of segmental coordination (Oullier et al. 2004).

When participants were informed about visual manipulation and were requested to resist it, they reduced body sway induced by the moving room. These results were similar to previous investigations (Aguiar et al. 2014; Barela et al. 2009, 2014; Freitas Junior and Barela 2004; Stoffregen et al. 2006). We found a reduction in the magnitude of body sway as well as in the amount of body sway that visual manipulation induced. Such a reduction was observed for participants from both groups. Therefore, knowledge about the visual manipulation and corresponding induced body sway, which implied that the participants became aware of their sway, produced changes in the postural control functioning and led to a less responsive system to the sensory manipulation. Despite this change, visual influences were not totally ignored by the participants and body sway was still induced by visual manipulation, even after 
they were aware that visual cues were unreliable. We can confirm this finding for both groups of the present study.

If we take into account that body sway was induced by visual manipulation, which suggested that the CNS cannot fully ignore available sensory cues, even when providing unreliable information, the coupling strength between the moving room and body sway was altered with awareness regarding the sensory manipulation according to task conditions. Coherence values changed dramatically from the "no instruction" to "resist" conditions, but only for those participants from the normal base of support group. Without any knowledge regarding visual manipulation, coherence values were approximately 0.9 and characterized by low variability. Differently, with knowledge regarding visual manipulation, the values of coherence dropped and revealed a much higher variability. Therefore, besides reducing the induced sway due to the visual manipulation, participants from the normal base of support group tried (although quite unsuccessfully) to avoid swaying with the moving room. The high variability observed for the coherence values indicates that each participant seems to try to avoid visual induction differently. When participants were unaware of visual manipulation, the coupling between visual information and body sway would reflect the CNS intrinsic dynamics, integrating visual cues into postural control, a consistent and similar process among participants. Differently, awareness about visual manipulation led to changes in the sensory-motor default coupling, in which participants tried to change the intrinsic CNS dynamics, each one taking different efforts in doing it and consequently achieving different success rates.

Participants from the reduced base of support group also showed high coherence values in the "no information" condition, but they were not able to change the coupling strength after being aware that visual manipulation was inducing body sway. They were able to reduce the amount of body sway, but still presented some change in the visual information and body sway coupling, which suggested that more demanding tasks require a stronger sensory-motor coupling. In this case, participants had no choice and/or possibilities to change the intrinsic dynamics employed by the CNS. Correspondent postural responses have been observed as visual stimulus characteristics, such as frequency (Dijkstra et al. 1994), amplitude, and velocity (Barela et al. 2014; Jeka et al. 2008), change. However, changes in postural control might not only take place due to simply corresponding visual stimuli characteristics; they may also be imposed by other factors, such as intention (Barela et al. 2009; Stoffregen et al. 2006). Adaptive postural control properties have been discussed and even modeled (Schöner et al. 1998). This is a key feature of postural control functioning, which provides advantages in such sensory-motor tasks (Jeka et al. 2008). However, our results indicate that adaptive mechanisms may be overcome by task and/or condition demands.

Finally, this study advances our understanding in an important aspect related to attention and the coupling between visual information and body sway. Before being aware of the visual manipulation and body sway induced by such manipulation, the concomitant task did not change the coupling strength (Fig. 2-no information) or the visual influence (Fig. 3a-no information). On the other hand, when participants from the normal base of support group had a chance to change the coupling strength due to the awareness of visual manipulation (Fig. 2a-information, normal support group), the concomitant task influenced coupling strength, making coherence values approach to the observed ones in the no information condition. Therefore, changes in the influence of the visual stimulus might occur due to information and/or the request to resist motion (Barela et al. 2009; Stoffregen et al. 2006), but such a change requires attentional efforts that might not be possible.

In sum, we suggest that the coupling between sensory information and motor activity is achieved by the CNS with very low or even no attentional requirements. None of the participants were aware that body sway had been induced, corroborating previous results involving adults (Barela et al. 2014; Freitas Junior and Barela 2004) and children (Rinaldi et al. 2009). Such a lack of awareness is also a strong indication of the low level of involvement of the higher centers of the CNS and that it is important in avoiding any overloading of the higher centers when performing low-level tasks, such as using sensory cues to perform motor activity. This clever functionality, however, might lead to the use of unreliable information.

\section{References}

Aguiar SA, Gramani-Say K, Lopes AG, Barela JA (2014) Dual task interferes with sensorimotor coupling in postural control. Psychol Neurosci 7:593-599. doi:10.3922/j.psns.2014.4.19

Andersson G, Hagman J, Talianzadeh R, Svedberg A, Larsen HC (2002) Effect of cognitive load on postural control. Brain Res Bull 58:135-139. doi:10.1016/S0361-9230(02)00770-0

Barela JA, Jeka JJ, Clark JE (2003) Postural control in children: coupling to dynamic somatosensory information. Exp Brain Res 150:434-442. doi:10.1007/s00221-003-1441-5

Barela AMF, Barela JA, Rinaldi NM, de Toledo DR (2009) Influence of imposed optic flow characteristics and intention on postural responses. Mot Control 13:119-129

Barela JA, Weigelt M, Polastri PF, Godoi D, Aguiar SA, Jeka JJ (2014) Explicit and implicit knowledge of environment states induce adaptation in postural control. Neurosci Lett 566:6-10. doi:10.1016/j.neulet.2014.02.029

Bucci MP, Ajrezo L, Wiener-Vacher S (2015) Oculomotor tasks affect differently postural control in healthy children. Int J Dev Neurosci 46:1-6. doi:10.1016/j.ijdevneu.2015.05.008

Dijkstra TM, Schöner G, Giese MA, Gielen CC (1994) Frequency dependence of the action-perception cycle for postural control 
in a moving visual environment: relative phase dynamics. Biol Cybern 71:489-501

Freitas Junior PB, Barela JA (2004) Postural control as a function of self- and object-motion perception. Neurosci Lett 369:64-68. doi:10.1016/j.neulet.2004.07.075

Horak FB, Macpherson JM (1996) Postural orientation and equilibrium. In: Rowell LB, Shepard JT (eds) Handbook of physiology. Oxford University Press, New York, pp 255-292

Jeka JJ, Ribeiro P, Oie K, Lackner JR (1998) The structure of somatosensory information for human postural control. Mot Control $2: 13-33$

Jeka JJ, Oie KS, Kiemel T (2008) Asymmetric adaptation with functional advantage in human sensorimotor control. Exp Brain Res 191:453-463. doi:10.1007/s00221-008-1539-x

Nashner LM (1981) Analysis of stance posture in humans. In: Towe AL, Luschei ES (eds) Motor coordination (handbook of behavioral neurology), vol 5., Plenum PressNew York, NY, pp 527-565

Oullier O, Bardy BG, Stoffregen TA, Bootsma RJ (2002) Postural coordination in looking and tracking tasks. Hum Mov Sci 21:147-167

Oullier O, Bardy BG, Stoffregen TA, Bootsma RJ (2004) Task-specific stabilization of postural coordination during stance on a beam. Mot Control 8:174-187

Polastri PF, Barela JA, Kiemel T, Jeka JJ (2012) Dynamics of intermodality re-weighting during human postural control. Exp Brain Res 223:99-108. doi:10.1007/s00221-012-3244-z

Prado JM, Stoffregen TA, Duarte M (2007) Postural sway during dual tasks in young and elderly adults. Gerontology 53:274-281. doi: $10.1159 / 000102938$

Prioli AC, Freitas Junior PB, Barela JA (2005) Physical activity and postural control in the elderly: coupling between visual information and body sway. Gerontology 51:145-148. doi:10.1159/000083984
Prioli AC, Cardozo AS, Freitas Junior PB, Barela JA Jr. (2006) Task demand effects on postural control in older adults. Hum Mov Sci 25:435-446. doi:10.1016/j.humov.2006.03.003

Remaud A, Boyas S, Caron GAR, Bilodeau M (2012) Attentional demands associated with postural control depend on task difficulty and visual condition. J Mot Behav 44:329-340. doi:10.108 0/00222895.2012.708680

Rinaldi NM, Polastri PF, Barela JA (2009) Age-related changes in postural control sensory reweighting. Neurosci Lett 467:225229. doi:10.1016/j.neulet.2009.10.042

Schöner G, Dijkstra TMH, Jeka JJ (1998) Action-perception patterns emerge from coupling and adaptation. Ecol Psychol 10:323-346. doi:10.1207/S15326969eco103\&4_9

Siu KC, Catena RD, Chou LS, van Donkelaar P, Woollacott MH (2008) Effects of a secondary task on obstacle avoidance in healthy young adults. Exp Brain Res 184:115-120. doi:10.1007/ s00221-007-1087-9

Stoffregen TA, Hove P, Schmit J, Bardy BG (2006) Voluntary and involuntary postural responses to imposed optic flow. Mot Control 10:24-33

Streepey JW, Angulo-Kinzler RM (2002) The role of task difficulty in the control of dynamic balance in children and adults. Hum Mov Sci 21:423-438

Toledo DR, Barela JA (2014) Age-related differences in postural control: effects of the complexity of visual manipulation and sensorimotor contribution to postural performance. Exp Brain Res 232:493-502. doi:10.1007/s00221-013-3756-1

Viana AR, Razuk M, Freitas Junior PB, Barela JA (2013) Sensorimotor integration in dyslexic children under different sensory stimulations. PLoS ONE 8:e72719. doi:10.1371/journal.pone.0072719 